\title{
MINIMALLY INVASIVE TREATMIENT OF ABDOMINAL LYMPHOCELE: A REVIEW OF CONTEMIPORARY OPTIONS AND HOW TO APPROACH THEM
}

\author{
F. Khorshidi, B.S. Majdalany, G. Peters, A.N. Tran, J. Shaikh, \\ R.P. Liddell, J.C. Perez Lozada, N. Kokabi, N. Nezami
}

Department of Radiology (FK), Tabriz University of Medical Sciences, Tabriz, Iran; Division of Interventional Radiology and Image-Guided Medicine (BSM,GP,ANT,JS,NK,NN), Department of Radiology and Imaging Sciences, Emory University School of Medicine, Atlanta, GA; Division of Vascular and Interventional Radiology (RPL), The Russell H. Morgan Department of Radiology and Radiological Science, The Johns Hopkins Medical Institutions, Baltimore, MD; Section of Vascular and Interventional Radiology (JCPL), Department of Radiology and Biomedical Imaging, Yale University School of Medicine, New Haven, CT; Division of Vascular and Interventional Radiology (NN), Department of Diagnostic Radiology and Nuclear Medicine, University of Maryland School of Medicine, Baltimore, MD, USA

\section{ABSTRACT}

Lymphoceles are lymphatic fluid collections resulting from lymphatic vessel disruption after surgery or trauma. They are most often described following retroperitoneal surgeries such as cystectomies, prostatectomies, renal transplants, and gynecologic surgeries. Most lymphoceles are asymptomatic and resolve spontaneously without treatment. If persistent, they can become infected or exert mass effect on adjacent structures causing pain, urinary, or lower limb edema particularly for lymphoceles in the pelvis Symptomatic lymphoceles should be treated to relieve symptoms and prevent functional compromise of vital adjacent structures.

Although surgery has been traditionally accepted as the gold standard treatment, advances in imaging and interventional technology allow for less invasive, percutaneous treatment. A vailable minimally invasive treatment options include percutaneous aspiration, catheter drainage, sclerotherapy, and lymphangiography with lymphatic embolization. A review of these treatment options and a suggested algorithm for managing lymphoceles is presented.

Keywords: lymphocele, post-operative complication, lymphangiography, lymphatic embolization, treatment algorithm, sclerotherapy

Lymphocele is a collection of lymphatic fluid within a fibrotic capsule (Fig. 1). It was first reported in 1950 by Kobayashi and Inoue as a complication of pelvic lymphadenectomy for gynecologic malignancy using the term lymphocyst (1). This term, however, does not appropriately describe lymphoceles as they are pseudocysts that lack an epithelial lining $(2,3)$.

Lymphatic fluid lacks platelets and contains reduced concentrations of clotting factors decreasing the possibility of thrombosis after lymphatic disruption and allowing for lymphocele development (4). The incidence of postoperative lymphocele ranges between $8 \%$ 


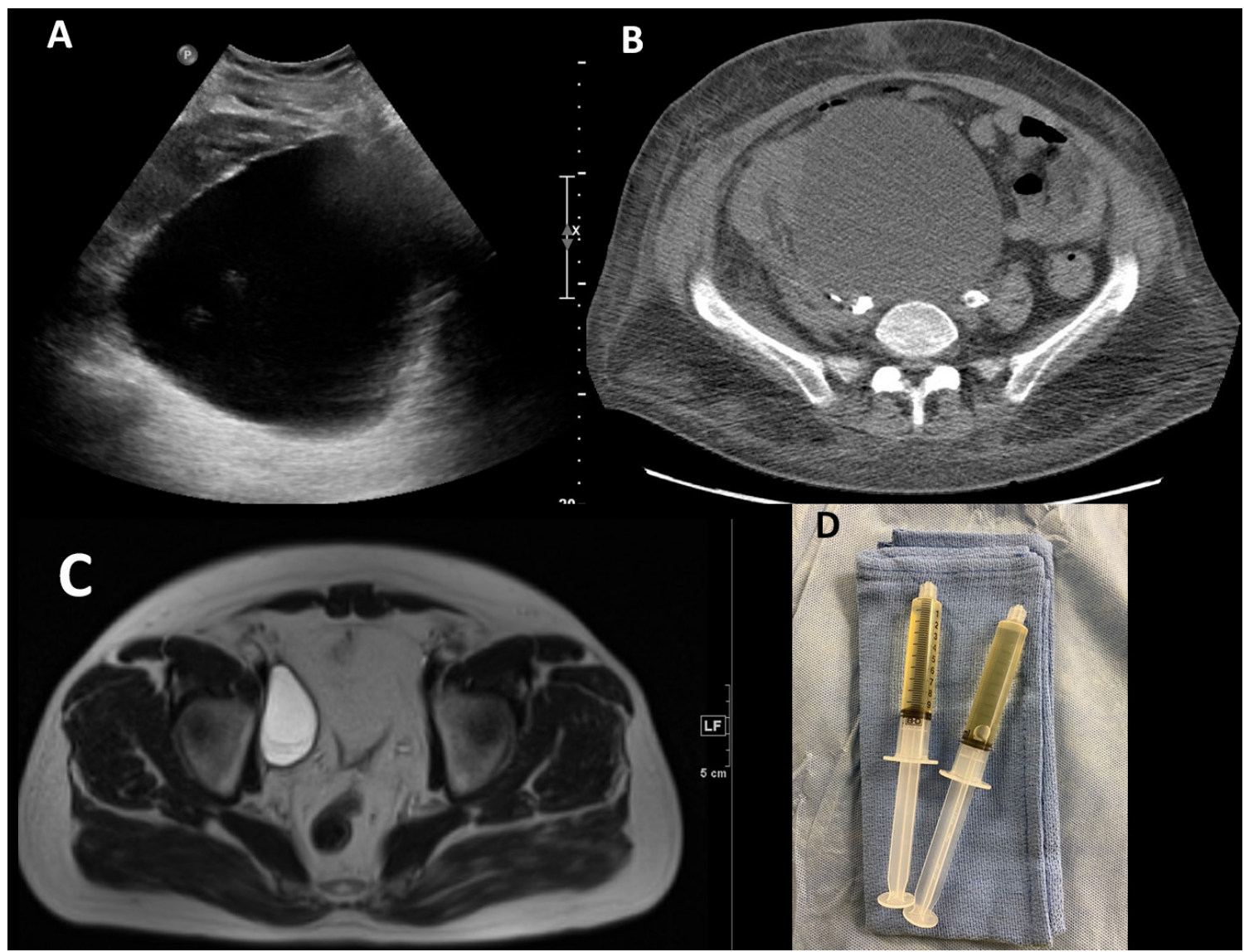

Fig. 1. Imaging findings of lymphocele. $A$. Transverse ultrasonographic view of the pelvis reveals an anechoic collection with an imperceptible wall, representing post-surgical lymphocele. B. Axial CT shows the same lymphocele, which has simple fluid attenuation. C. Axial T2 MRI sequence shows a pelvic lymphocele in a different patient with high signal representing simple fluid. D. Gross picture of the aspirated sample fluid which is thin, light yellow, and without odor.

and $48 \%$ (2). They are more common in extraperitoneal surgeries, often as a complication of cystectomies and prostatectomies when lymph node dissection is required with urogenital malignancies, or renal transplant surgery (3,5-10). Lymphocele has also been reported after vascular surgery $(11,12)$, spinal surgery $(13,14)$, and trauma (15). Lymphoceles often develop 3 to 8 weeks after surgery; however, rare occurrences up to 8 years later have been described $(10,11)$.

Most lymphoceles are small, asymptomatic, and incidentally diagnosed (Figs. $2 A-D$ ). They may become infected or when large enough, compress adjacent structures causing pain, abdominal fullness, leg swelling, constipation, deep venous thrombosis or renal function deterioration in the setting of urologic procedures (16-20).

The majority of lymphoceles do not require intervention and resolve spontaneously $(8,10,17,21)$. In approximately $4-7 \%$ of patients they persist (2). When lymphoceles persist and cause symptoms, intervention becomes essential $(8,18)$. Minimally invasive therapeutic options for symptomatic postoperative lymphoceles include image-guided needle aspiration, percutaneous catheter 


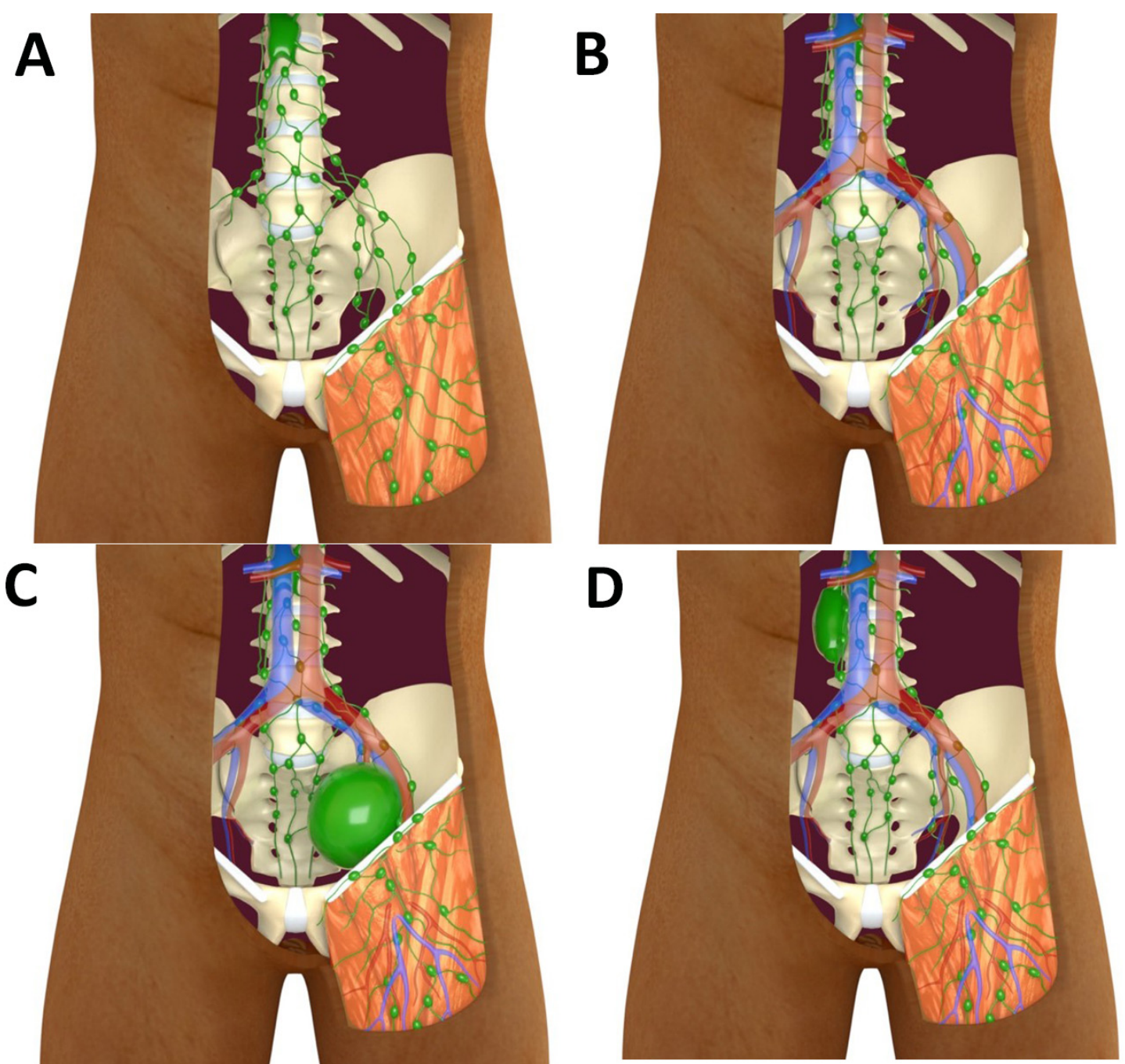

Fig. 2. Schematic pictures of lymphatic system and lymphoceles. $A$ and B. Normal lymphatic system shown alone $(A)$ and in relation to the abdominopelvic vasculatures $(B)$ in the abdomen and pelvis, C. Lymphocele in the pelvis. D. Retroperitoneal lymphocele.

drainage with or without the use of sclerosing agents, percutaneous image-guided lymphatic ligation (PILL), and lymphangiography with embolization. If percutaneous treatments are unsuccessful, laparoscopic or open surgical marsupialization is performed (2,21-24). The purpose of this review is to highlight the treatments of lymphoceles and develop a treatment guide for patients who are likely to encounter potentially problematic lymphoceles.

\section{TREATMENTS}

\section{Simple Needle Aspiration}

In the late 1970 s and early 1980 s, percutaneous image-guided simple needle aspiration was used for lymphocele treatment. However, high rates of recurrence $(80-90 \%)$ and infection (25-50\%) led to discontinuation of this practice $(18,25)$. Currently, needle aspiration is indicated for diagnostic purposes and temporary symptomatic relief $(25,26)$. Lymphatic fluid is typically clear and light yellow 


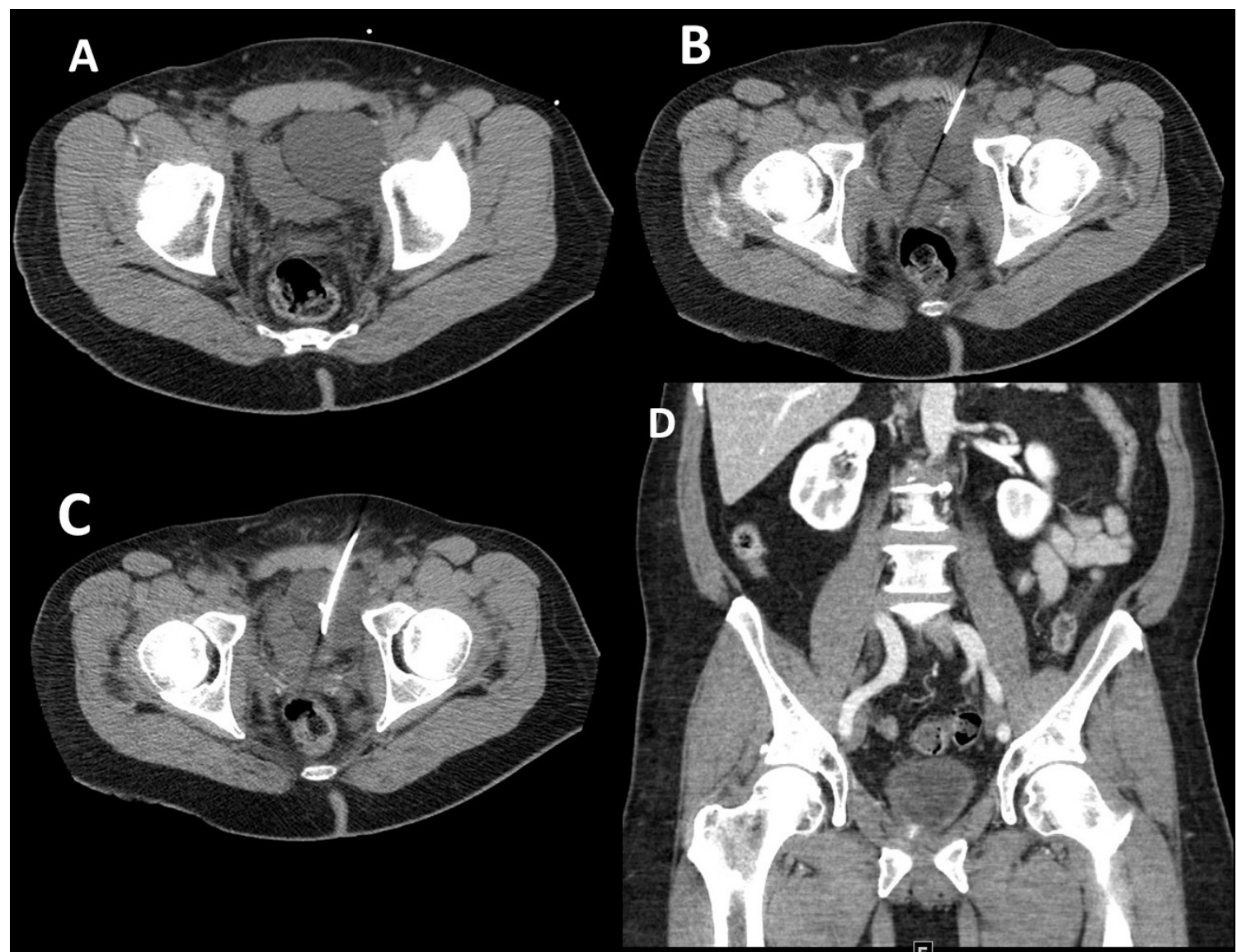

Fig. 3. Drain placement. CT-guided drain placement for lymphocele in a patient with history of prostate cancer who underwent prostatectomy and lymphadenectomy. A. Axial CT imaging shows a left pelvic collection which displaces the bladder posteriorly B. Axial CT imaging, intraprocedural during placement of the introducer. $C$. Final $C T$ scan demonstrates the location of the drain. D. Coronal CT image post-treatment reveals resolution of the lymphocele after 13 days.

in color. Testing of the aspirate (Fig. 1) should include fluid creatinine and cell count and differential, with low creatinine and preponderance of lymphocytes $(>70 \%)$ indicative of a lymphocele.

\section{Percutaneous Catheter Drainage}

In 1983, Aronowitz and Kaplan performed the first successful percutaneous catheter drainage (PCD) of a post-operative lymphocele (27). PCD is a simple method in which a catheter is inserted into the lymphocele using ultrasound, computed tomography (CT), or fluoroscopic guidance. As shown in
Fig. 3, the catheter is left indwelling to allow continuous drainage $(25,27,28)$. It is usually removed when the daily output is less than $\mathbf{1 0}$ $\mathrm{mL}$ and there is collapse of the lymphocele cavity on the imaging (28-30).

If clinical symptoms or high-volume catheter output persist, alternative treatments may be necessary $(28,30)$. With prolonged drainage, success rates of $79-100 \%$ have been reported (30). However, studies that provide data on the rate of recurrence show that PCD is associated with 50-63.6\% recurrence rate $(6,17)$. In addition, the lymphatic output may continue for as long as $\mathbf{4}$ months, potentially delaying the patient's further care plan and 
and increasing the risk of infection (29).

\section{Sclerotherapy}

Percutaneous transcatheter sclerotherapy is a low-risk image-guided procedure. After insertion of a catheter into the lymphocele and evacuating the fluid, contrast is injected into the cavity to exclude extravasation and communication with vital structures and vasculature. The contrast is then drained to estimate the volume of sclerosing agent, which is subsequently instilled into the lymphocele $(2,25,31)$.

The procedure is usually performed on an outpatient basis at weekly intervals $(2,32,33)$ or every other day $(25,31,34)$. Success is defined as complete collapse of the cavity based on imaging and daily output from the drainage catheter of less than $10 \mathrm{~mL}$ (2). A failed procedure is defined by persistent drainage or when any complication is encountered that prompts a decision to cease sclerotherapy treatment (2).

Percutaneous transcatheter sclerotherapy was first described as an effective first-line treatment in 1983. Two cases of post-operative lymphocele were sclerosed using PovidoneIodine as the sclerosing agent (35). The proposed mechanism of action is irritation of the lymphocele's walls, which induces local inflammation and fibrosis of the lymphatic channels, obliterating the lymphatic leak $(2,33)$. Additional agents that have subsequently been used for lymphocele sclerotherapy include ethanol, ampicillin, tetracycline, doxycycline, bleomycin, sodium azetroate, sodium tetradecyl sulfate, sodium morhuate, talc, and fibrin (31). Due to lack of robust scientific evidence comparing the effectiveness and efficacy of the individual sclerosing substances, choosing one over another depends on availability and the operator's personal preferences and experience $(2,31,33)$.

It has been proposed that sclerotherapy is more likely to fail in lymphoceles larger than 150 to $200 \mathrm{~mL}(2,25)$. The reported success rate of sclerotherapy ranges between 62$100 \%(2,17,25,31-34)$, with recurrence rates between $3-38 \%(6,33)$. Infection has been reported in up to $9 \%$ (32). However, studies that used prophylactic antibiotics reported no infection (34).

\section{Lipiodol Lymphangiography}

Lymphangiography (LG) is a valuable tool for detection of post-operative lymphatic leak. It has also proven effective in treating lymphatic leakage because the injected ethiodized oil (Lipiodol , Guerbet, France) seems to induce an inflammatory reaction at the site of lymph outflow which leads to closure of the leak (22).

There are two methods of LG: pedal and intranodal (36,37). Conventional pedal LG involves the isolation and cannulation of a dorsal pedal lymphatic vessels with very slow injection of lipiodol and serial radiographs to detect the progression of ethiodized oil and evaluate for a lymphatic leak. It is technically challenging, time-consuming and requires a cutdown to expose the tiny pedal lymphatics. Alternatively, intranodal LG relies on ultrasound guidance to directly access the inguinal lymph node for injection of lipiodol (Figs. 4 and 5). Intranodal LG is technically easier, widely available, has less morbidity, and does not require a slow infusion pump $(36,37)$.

The time between LG and lymphatic leak cure is reported to be between 7 and 14 days $(22,36)$. LG success may depend on the volume of lymphatic drainage per day. In a retrospective analysis of 50 patients with lymphatic leakage in the form of chylothorax, chylous ascites, lymphocele, and lymphatic fistulae, those with drainage volumes of less than $500 \mathrm{~mL} /$ day had a two-fold higher success rate $(70 \%)$ compared to patients with more than $500 \mathrm{~mL} /$ day (35\%) (22).

There are limited data on therapeutic success of LG in the treatment of lymphocele with some even showing discouraging results. In the previously mentioned study, LG therapeutic success in lymphocele cases was only $50 \%$, but other studies have suggested that higher volumes of ethiodized oils have more therapeutic benefit $(22,38)$.

A retrospective review of 22 pedal lipiodol lymphangiograms (18 monopedal, 2 


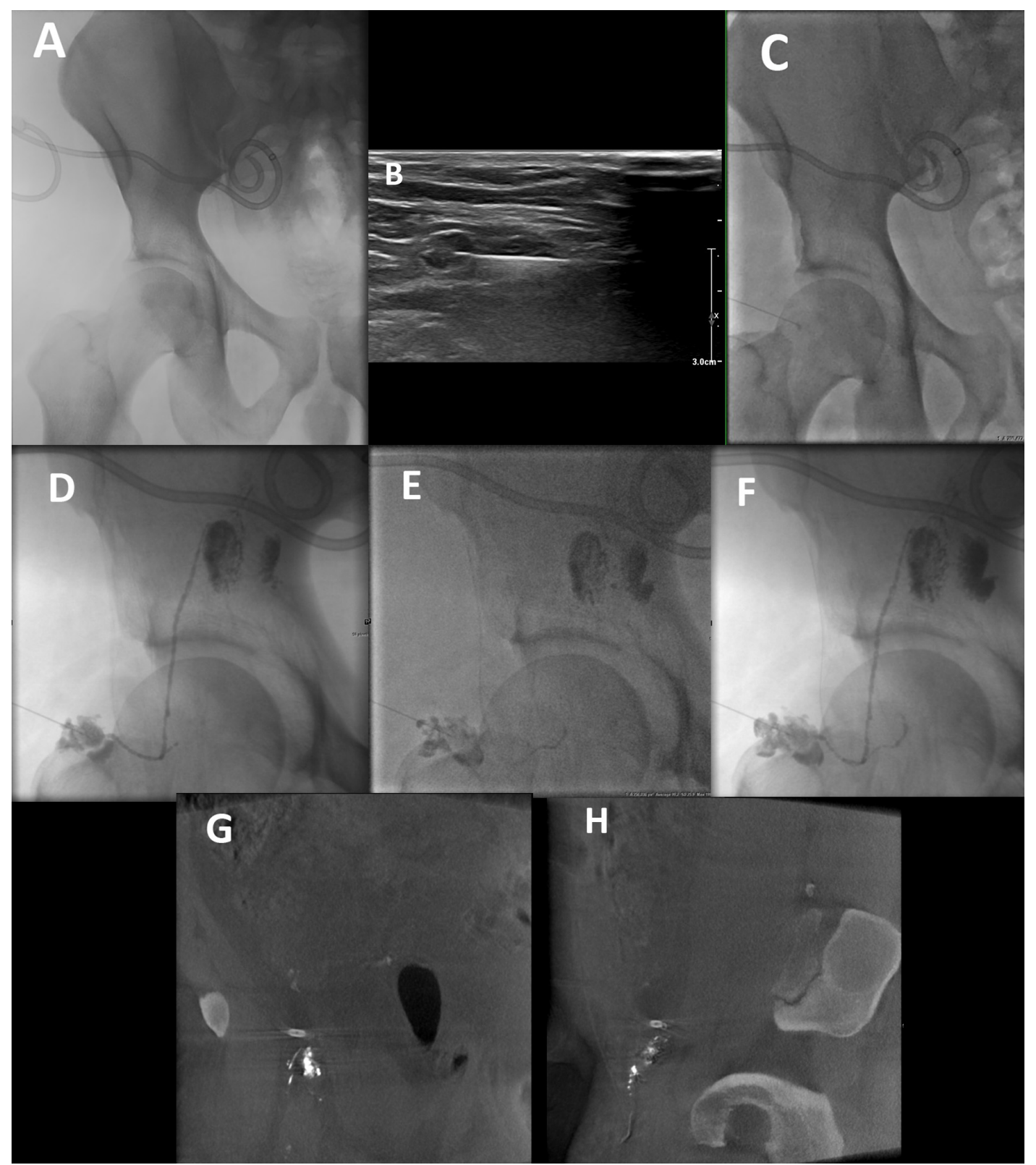

Fig. 4. Single needle technique. Lymphangiogram and n-butl cyanoacrylate (NBCA) glue embolization. 39-year-old African-American male who was diagnosed with muscle invasive bladder cancer and underwent radical cystectomy and neobladder creation, without neoadjuvant chemotherapy. The patient presented with reaccumulation of fluid in the pelvis after 2 months. A. Drain in place on the scout image. Lymphangiogram was performed by accessing a right inguinal lymph node using a $25 \mathrm{G}$ needle under ultrasound and fluoroscopic guidance $(B$ and $C$ ) with lipiodol injection delineating the lymph chain communicating with the right pelvic lymphocele and leak (D). E. Subsequently, Dextrose 5\% injection was used to clear the lipiodol and prevent premature polymerization of the glue. F. A 5:1 ratio of lipiodol to NBCA glue was used to embolize the inflow lymph vessel and allow polymerization of glue at the site of the lymphatic injury, successfully sealing the extravasation. $G \& H$. Coronal and sagittal views of cone beam CT of the lymphocele and embolized glue. The drain output decreased to less than $10 \mathrm{~mL}$ after 3 days and the drain was removed. 


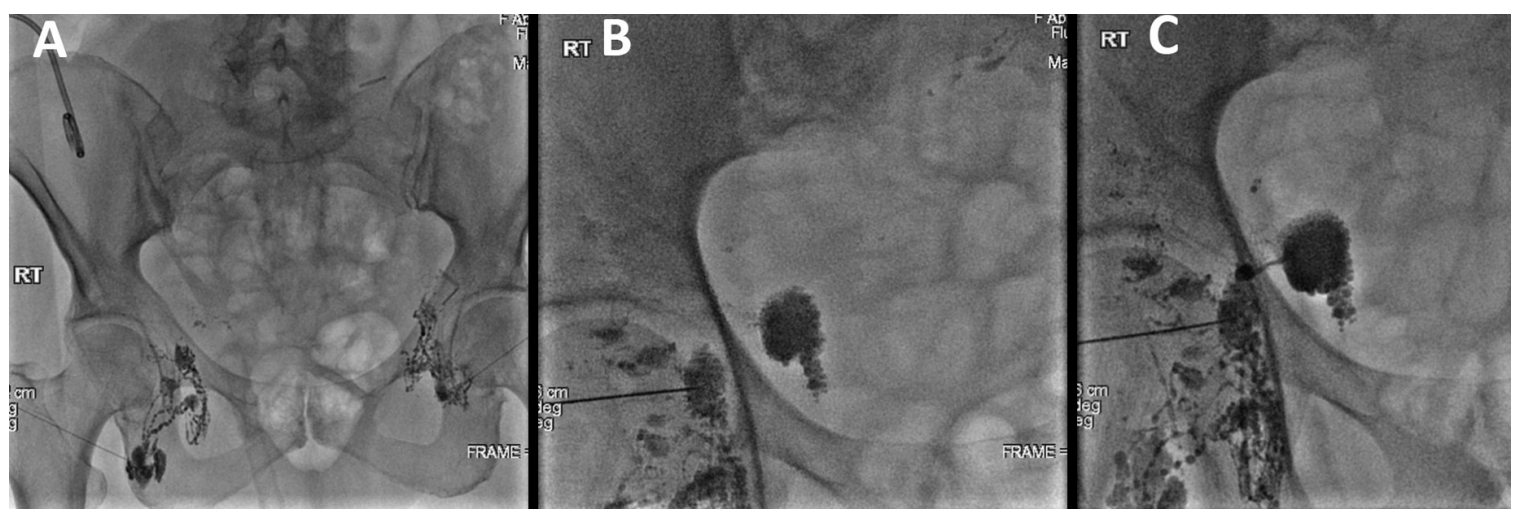

Fig. 5. Double needles technique. A. The lymphatics are opacified by injecting lipiodol through the groin lymph node. $B$. The closest lymph node to the leak is identified and accessed by another needle (22 G Chiba needle). C. Subsequently, the leak is embolized using a 2:1 ratio of lipiodol to NBCA glue.

bipedal) in patients with post-operative lymphatic leak, LG not only localized the leak in $75 \%$ of patients, but also closed the leak in $55 \%$ of patients (39).

\section{Glue Embolization}

N-butyl cyanoacrylate (NBCA) gluebased embolization with lipiodol LG is a relatively new method for treating lymphatic leaks refractory to conservative treatment and sclerotherapy $(23,40)$. After identifying the lymphatic leak using lipiodol LG, inflow lymph nodes or supplying lymphatic vessels are targeted percutaneously. Under fluoroscopic or C-arm cone-beam computed tomography (CBCT) guidance, NBCA glue mixed with lipiodol is injected at the puncture site to treat the downstream lymphatic leak through the access needle (Figs. 4A-H) or through a second needle placed closer to the leakage site (Figs. 5A-C) (23,40-42).

The success rate of NBCA embolization in treating postoperative lymphoceles has been reported as $80-100 \%(23,40-43)$. Lymphoceles with multiple leaking vessels are more likely to fail initial treatment and require repeated embolization with or without sclerotherapy to occlude the leak $(23,41)$.

Complications related to $\mathrm{LG}$ and adjunctive embolization may include mild transient pain or leg edema which can be managed conservatively $(23,42)$. A serious complication related to the use of higher lipiodol volumes is pulmonary lipiodol embolization. This usually does not produce any clinical symptoms unless there is an underlying cardiopulmonary disorder such as pulmonary insufficiency or rightto-left cardiac shunt (36).

\section{Open and Laparoscopic Surgical Marsupialization}

Operative treatment of symptomatic lymphocele consists of lymphocelectomy with creation of a large internal window for intraperitoneal fluid drainage (3). Open surgery and internal marsupialization had historically been accepted as the gold standard treatment. Long hospital stays with increased morbidity and mortality due to surgery precludes use in all patients (31). Recently, laparoscopic marsupialization has appeared as an alternative surgical method. It is less hostile to the abdominal wall with quicker recovery, favorable cosmetics, reduced blood loss, low post-operative morbidity and shorter hospital stays by at least $50 \%$ compared to open surgery $(3,6)$. A success rate of $95 \%$ has been described for laparoscopic surgery in two more recent series (33).

Open surgery is associated with a recurrence rate of $16 \%$, while the rate of recurrent lymphocele formation after laparoscopic fenestration is $8 \%$ (6). During laparoscopic surgery, conversion to open surgery may be 
required in $12 \%$ (6). Laparoscopic surgery has an overall complication rate of $14 \%$ compared with $30 \%$ for open surgery (6).

\section{Percutaneous Image-Guided Lymphatic Ligation}

There are a few other less investigated methods for treating post-operative lymphocele. In percutaneous image-guided lymphatic ligation, the leak is defined with lipiodol LG. Under fluoroscopy, the responsible vessel is ligated percutaneously using a small skin incision (21). More studies are needed to confirm efficacy and safety of this method.

\section{DISCUSSION}

Lymphocele is an extraperitoneal or retroperitoneal lymph-filled collection that complicates $8 \%-48 \%$ of all surgeries $(2,31)$. Most lymphoceles are small, asymptomatic, incidentally diagnosed on routine imaging, and resolve without treatment $(2-6,8,10,33)$. Approximately 4-7\% of lymphoceles persist (2). These collections may lead to symptoms due to infection or compression of adjacent structures $(2,4,9,24)$.

Although there are multiple options available for treatment, limited management algorithms have been suggested to date (44). We have reviewed and evaluated variable approaches and developed a new treatment algorithm (Fig. 6) for managing lymphoceles. Although our approach is focused on abdominal lymphoceles, these approaches may be utilized for other lymphoceles (i.e., those after breast cancer operations).

Asymptomatic lymphoceles do not require any intervention and should be monitored for spontaneous regression using interval imaging. In a retrospective series including 521 patients who developed lymphocele after radical prostatectomy and pelvic lymph node dissection, $64 \%$ of lymphoceles persisting at 3 months after surgery became infected and required drainage (45). Thus, treatment is appropriate if symptoms appear during followup or when lymphoceles persist beyond 3 months post-surgery to prevent possible infection.

Percutaneous catheter drainage, despite being simple, economical and safe, is not a desirable method due to high recurrence rates (50-63.6\%) (17) and prolonged drainage duration which increases hospital stay, complicates wound care, and carries an infection rate of $20 \%(6,27)$. In a study of 11 patients with lymphocele, percutaneous drainage success rate was $78 \%$ with a drainage duration of 4120 days, however, $27 \%$ of cases developed secondary infection (29). In another study, overall success rate was reported to be $78 \%$; however, $56 \%$ of these cases recurred requiring repeat drainage, making the single drainage success rate only $25 \%$ (46).

Previous studies confirm that infected collections were more likely to resolve with PCD alone, since inflammatory changes in infected lymphocele render the lymphocele adhesive, which results in shorter drainage duration $(26,28,30,46)$. In a study of 23 lymphoceles, of which $43 \%$ were infected, percutaneous drainage had an $87 \%$ success rate with only $13 \%$ recurrence (30). We suggest percutaneous drainage be considered as a first-line treatment only for infected postoperative lymphoceles.

Instillation of sclerosing agents through drainage catheters is an attractive alternative because of its lower reported recurrence rates and shorter duration of therapy than percutaneous catheter drainage alone (17). Despite being less invasive, sclerotherapy has comparable success rates to surgery $(17,31,33)$. A prerequisite is that the integrity of the wall outlining the collection be intact without leaks to prevent intraperitoneal spillage of the sclerosing agent. This leakage may cause pain, peritoneal hemorrhage, and intravascular absorption resulting in toxicity (23).

Commonly used sclerosants include absolute ethanol, acetic acid, povidone-iodine, bleomycin, and minocycline (23). Scientific evidence has not proven any particular agent to be more effective than others; therefore, the choice is based on operator preferences and experience $(2,31,33)$. Ethanol is currently most commonly used and it is a simple and costeffective sclerosant for the treatment of 


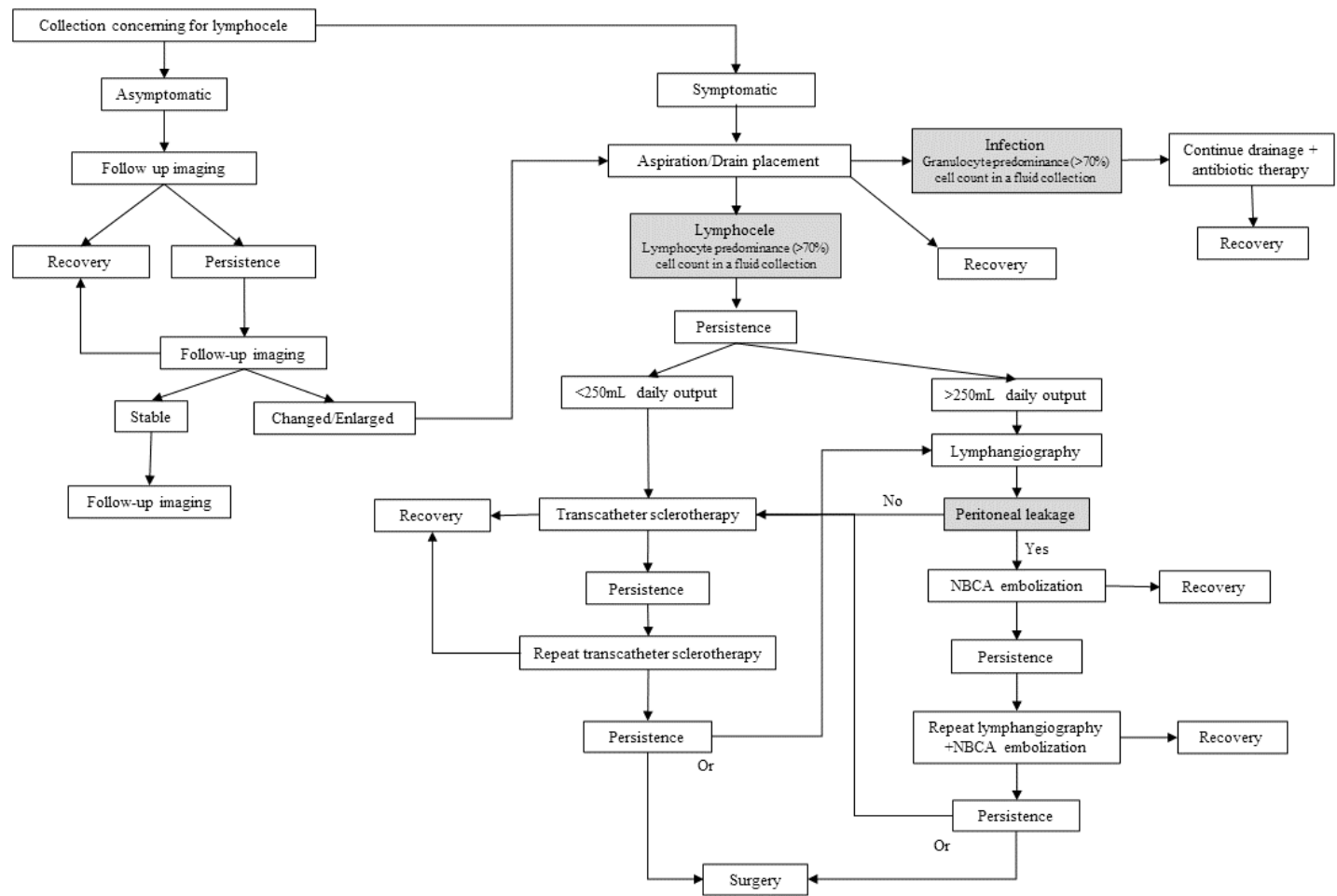

Fig. 6. A suggested algorithm to approach lymphocele treatment.

lymphocele $(25,40)$.

Sclerotherapy has a reported success rate of $62-100 \%(2,8,17,25,31-34)$, and the recurrence rate is between $3-38 \%(6,8,33)$. Most recurrences resolve with additional rounds of sclerosis (8). One factor that has been associated with the outcome of sclerotherapy is lymphocele size with larger cavities associated with lower success rates due to dilution of the sclerosant $(2,23)$.

In a study of 43 patients with post-surgical lymphoceles treated with sclerotherapy using a combination of povidone iodine, alcohol, and doxycycline, an overall $77 \%$ success rate was reported (2). There was a lower success rate with larger cavities and a significant difference $(p<.05)$ in the fluid volume at initial drainage between the failure group $(1,708$ $\mathrm{mL}$ ) and the success group (206 $\mathrm{mL})(2)$.

In a retrospective study of 52 patients with post-operative lymphocele, those with volumes smaller than $150 \mathrm{~mL}$ were treated by single-session ethanol sclerotherapy. For patients with a lymphocele volume of greater than $150 \mathrm{~mL}$, ethanol sclerotherapy was continued every other day until daily drainage reduced to below $10 \mathrm{~mL}$. The overall reported success rate was $92 \%$. All lymphoceles with volumes less than $150 \mathrm{~mL}$ were treated without any recurrence, while those with volumes larger than $150 \mathrm{~mL}$ had a $90 \%$ success rate with $10 \%$ recurrence $(25)$.

Lymphoceles with volumes higher than $150 \mathrm{~mL}$ should be scheduled for multiple session outpatient sclerotherapy until the drainage volume reduces to $10 \mathrm{~mL}$ per day. In case of failure or complications, other options such as adjuvant lymphangiography with embolization or laparoscopic surgery depending on availability and patient criteria should be considered.

NBCA glue-based embolization with lipiodol LG is a relatively novel method used for treating lymphatic leaks refractory to 
conservative treatment and sclerotherapy $(23,40,47)$. Available data are from studies with relatively small numbers of patients. Success rates are $80-100 \%(23,40-43)$. Lymphoceles with multiple leaking vessels are more likely to fail initial treatment and require repeated embolization with or without sclerotherapy to occlude the leak $(23,41)$.

In a retrospective analysis, nine patients with post-operative lymphocele were treated with LG and lymphatic embolization using NBCA glue mixed with Lipiodol oil in a ratio of 1:2 to $1: 9$ (41). Three patients that required repeated embolization had multiple lymphatic leaks. In another case series of 5 patients, post-operative lymphoceles were treated with lymphangiography and embolization (23). Two patients out of 5 had lymphoceles with multiple inflow vessels and initial procedures failed in both patients, prompting repeat embolization with adjunctive sclerotherapy (23).

Studies on glue embolization show promising results; however, because of the complex nature of the lymphatic system, injury to lymphatic vessels can be extensive. Depending on the invasiveness of the surgery, post-operative lymphatic leakage may be multifocal, requiring multiple procedures for complete occlusion of lymphatic leakage (41).

Larger patient cohorts and longer followup are required to verify the efficacy and outcomes of percutaneous lymphatic interventions in the management of post-operative lymphocele. This data is requisite for percutaneous intervention to be considered as the new gold standard first-line treatment option.

With the frequency of post-surgical iatrogenic lymphoceles, a proper treatment algorithm is required to reduce the serious sequelae of lymphoceles, with treatments ranging from minimally invasive drainage to open surgery. Prospective cohort studies that examine the benefits of each treatment would bolster the data supporting each treatment. Further studies should focus on statistically comparing these treatment methods through a systematic review and meta-analysis.

\section{CONFLICT OF INTEREST AND DISCLOSURE}

All authors declare that no financial conflict of interest exist.

\section{REFERENCES}

1. Kobayashi, $\mathrm{T}, \mathrm{S}$ Inoue: Pelvic lymphocyst. Clin. Obstet. Gynecol. 4 (1950), 91-95.

2. Mahrer, A, P Ramchandani, SO Trerotola, RD Shlansky-Goldberg, M Itkin:

Sclerotherapy in the management of postoperative lymphocele. J. Vasc. Interv. Radiol. 21 (2010), 1050-1053. 10.1016/j.jvir.2010.03.014.

3. Lima, ML, CA Cotrim, JC Moro, R Miyaoka, CA D'Ancona: Laparoscopic treatment of lymphoceles after renal transplantation. Int. Braz. J. Urol. 28 (2012), 215-221; discussion 21. 10.1590/s1677-55382012000200009.

4. Gomez, M, NT Tanner, RA Recavarren, J Ravenel, GA Silvestri: A 79-year-old woman with a right paratracheal mass. Chest 135 (2009), 1384-1388. 10.1378/chest.08-2228.

5. Ranghino, A, GP Segoloni, F Lasaponara, L Biancone: Lymphatic disorders after renal transplantation: New insights for an old complication. Clin. Kidney J. 8 (2015), 615622. $10.1093 / \mathrm{ckj} / \mathrm{sfv064}$.

6. Lucewicz, A, G Wong, VWT Lam, et al: Management of primary symptomatic lymphocele after kidney transplantation: A systematic review. Transplantation 92 (2011), :663-673. 10.1097/tp.0b013e31822a40ef.

7. Bzoma, B, J Kostro, A Dębska-Ślizień, et al: Treatment of the lymphocele after kidney transplantation: A single-center experience. Transplantation Proceedings 48 (2016), 16371640.

https://doi.org/10.1016/j.transproceed.2016.03 .025 .

8. Lee, HJ, CJ Kane: How to minimize lymphoceles and treat clinically symptomatic lymphoceles after radical prostatectomy. Curr. Urol. Rep. 15 (2014), 445. 10.1007/s11934-014-0445-y.

9. Stolzenburg, JU, I Kyriazis, E Liatsikos: Postoperative lymphocele formation after pelvic lymph node dissection at the time of radical prostatectomy should not be considered an inevitable consequence of the approach. Eur. Urol. 71 (2017), 159-60. 10.1016/j.eururo.2016.08.046.

10. Kim, HY, JW Kim, SH Kim, YT Kim, JH Kim: An analysis of the risk factors and 
management of lymphocele after pelvic lymphadenectomy in patients with gynecologic malignancies. Cancer Res. Treat. 36 (2004), 377-383. 10.4143/crt.2004.36.6.377.

11. Garrett, HE, Jr., JW Richardson, HS Howard, HE Garrett: Retroperitoneal lymphocele after abdominal aortic surgery. $J$. Vasc. Surg. 10 (1989), 245-253.

12. Uchinami, M, K Morioka, K Doi, et al: Retroperitoneal laparoscopic management of a lymphocele after abdominal aortic surgery: A case report. J. Vasc. Surg. 42 (2005), 552555. 10.1016/j.jvs.2005.04.029.

13. Hey, HWD, KL Wong, AR Gatam, JL Lim, HK Wong: Delayed lymphocele formation following lateral lumbar interbody fusion of the spine. Eur. Spine J. 26 (2017), 36-41. 10.1007/s00586-016-4678-y.

14. Patel, AA, WR Spiker, MD Daubs, et al: Retroperitoneal lymphocele after anterior spinal surgery. Spine (Phila Pa 1976) 33 (2008), E648-652. 10.1097/BRS.0b013e31817c6ced.

15. Sato, R, S Kobayashi, K Uchida, $O$ Yokoyama, H Baba: Retroperitoneal lymphocele associated with lumbar spine fracture: Report of an unusual case. Spinal Cord 43 (2005), 687-690. 10.1038/sj.sc.3101775.

16. Kawaguchi, S, T Nohara, T Shima, et al: Massive ascites in a renal transplant patient after laparoscopic fenestration of a lymphocele. Case Rep. Transplant. 2016 (2016), 7491627. 10.1155/2016/7491627.

17. Góes Junior, AM, SA Jeha: Idiopathic lymphocele: A possible diagnosis for infraclavicular masses. Case Rep. Surg. 2012 (2012), 593028. 10.1155/2012/593028.

18. Heyman, JH, DE Orron, E Leiter: Percutaneous management of postoperative lymphocele. Urol. 34 (1989), 221-224. 10.1016/0090-4295(89)90378-6.

19. Metcalf, KS, KR Peel: Lymphocele. Ann. Roy. Coll. Surg. 75 (1993), 387-392.

20. Nghiem, DD, I Beckman: Intraperitoneal catheter drainage of lymphocele: An outpatient procedure. Transpl. Int. 18 (2005), 721-723. 10.1111/j.1432-2277.2005.00137.x.

21. Todokoro, T, D Furniss, K Oda, et al: Effective treatment of pelvic lymphocele by lymphaticovenular anastomosis. Gynecol. Oncol. 128 (2013), 209-214. 10.1016/j.ygyno.2012.11.014.

22. Alejandre-Lafont, E, C Krompiec, WS Rau, GA Krombach: Effectiveness of therapeutic lymphography on lymphatic leakage. Acta Radiologica 52 (2011), 305-311. 10.1258/ar.2010.090356.

23. Baek, Y, JH Won, SJ Chang, et al: lymphatic embolization for the treatment of pelvic lymphoceles: Preliminary experience in five patients. J. Vasc. Interv. Radiol. 27 (2016), 1170-1176. 10.1016/j.jvir.2016.04.011.

24. Varga, Z, A Hegele, P Olbert, R Hofmann, AJ Schrader: Laparoscopic peritoneal drainage of symptomatic lymphoceles after pelvic lymph node dissection using methylene blue instillation. Urol. Int. 76 (2006), 335-338. 10.1159/000092058.

25. Akhan, O, M Karcaaltincaba, MN Ozmen, D Akinci, D Karcaaltincaba, A Ayhan: Percutaneous transcatheter ethanol sclerotherapy and catheter drainage of postoperative pelvic lymphoceles. Cardiovasc. Intervent. Radiol. 30 (2007), 237240. 10.1007/s00270-006-0180-y.

26. Shokeir, AA, TA el-Diasty, Ghoneim MA. Percutaneous treatment of lymphocele in renal transplant recipients. J. Endourol. 7 (1993), 481-485. 10.1089/end.1993.7.481.

27. Aronowitz, J, AL Kaplan: The management of a pelvic lymphocele by the use of a percutaneous indwelling catheter inserted with ultrasound guidance. Gynec. Onc. 16 (1983), 292-295. https://doi.org/10.1016/00908258(83)90105-1.

28. Alago, W, Jr., A Deodhar, H Michell, et al: Management of postoperative lymphoceles after lymphadenectomy: Percutaneous catheter drainage with and without povidoneiodine sclerotherapy. Cardiovasc. Intervent. Radiol. 36 (2013), 466-471. 10.1007/s00270012-0375-3.

29. White, M, PR Mueller, JT Ferrucci, et al: Percutaneous drainage of postoperative abdominal and pelvic lymphoceles. Am. J. Roentgenol. 1445 (1985), 1065-1069. 10.2214/ajr.145.5.1065.

30. Kim, JK, YY Jeong, YH Kim, et al: Postoperative pelvic lymphocele: treatment with simple percutaneous catheter drainage. Radiology 212 (1999), 390-394. 10.1148/radiology.212.2.r99au12390.

31. Karcaaltincaba, M, O Akhan: Radiologic imaging and percutaneous treatment of pelvic lymphocele. Eur. J. Radiol. 55 (2005), 340-354. 10.1016/j.ejrad.2005.03.007.

32. Zuckerman, DA, TD Yeager: Percutaneous ethanol sclerotherapy of postoperative lymphoceles. Am. J. Roentgenol. 169 (1997); 
433-437. 10.2214/ajr.169.2.9242748.

33. Fernandes, AS, A Costa, R Mota, V Paiva:

Bleomycin sclerotherapy for severe

symptomatic and persistent pelvic

lymphocele. Case Rep. Obstet. Gynecol. 2014

(2014), 624803. 10.1155/2014/624803.

34. Sawhney, R, HB D'Agostino, S Zinck, et al:

Treatment of postoperative lymphoceles with percutaneous drainage and alcohol sclerotherapy. J. Vasc. Interv. Radiol. 7 (1996), 241-245. 10.1016/s10510443(96)70769-8.

35. Teruel, JL, EM Escobar, C Quereda, T Mayayo, J Ortuño: A simple and safe method for management of lymphocele after renal transplantation. J. Urol. 130 (1983), 10581059. https://doi.org/10.1016/S00225347(17)51683-3.

36. Iwai, $\mathrm{T}, \mathrm{J}$ Uchida, Y Matsuoka, et al: Experience of lymphangiography as a therapeutic tool for lymphatic leakage after kidney transplantation. Transplant. Proc. 50 (2018), 2526-2530.

10.1016/j.transproceed.2018.03.095.

37. Lee, EW, JH Shin, HK Ko, et al: Lymphangiography to treat postoperative lymphatic leakage: A technical review. Korean J. Radiol. 15 (2014), 724-732. 10.3348/kjr.2014.15.6.724.

38. Jardinet, T, HV Veer, P Nafteux, et al: Intranodal lymphangiography with high-dose ethiodized oil shows efficient results in patients with refractory, high-output postsurgical chylothorax: A retrospective study. Am. J. Roentgenol. 217 (2021), 433438. 10.2214/AJR.20.23465.

39. Kos, S, H Haueisen, U Lachmund, T Roeren: Lymphangiography: Forgotten tool or rising star in the diagnosis and therapy of postoperative lymphatic vessel leakage. Cardiovasc. Intervent. Radiol. 30 (2007), 968973. 10.1007/s00270-007-9026-5.

40. Yildirim, IOO, T Pişkin, E Duman, et al: A novel technique in the treatment of lymphoceles after renal transplantation: $\mathrm{C}$ Arm cone beam CT-guided percutaneous embolization of lymphatic leakage after lymphangiography. Transplantation 102 (2018), 1955-1960. $10.1097 /$ tp.0000000000002268.
41. Chu, HH, JH Shin, JW Kim, et al: Lymphangiography and lymphatic embolization for the management of pelvic lymphocele after radical prostatectomy in prostatic cancer. Cardiovasc. Intervent. Radiol. 42 (2019), 873-879. 10.1007/s00270019-02209-6.

42. Hur, S, JH Shin, IJ Lee, et al: Early experience in the management of postoperative lymphatic leakage using lipiodol lymphangiography and adjunctive glue embolization. J. Vasc. Interv. Radiol. 27 (2016), 1177-1186.e1. 10.1016/j.jvir.2016.05.011.

43. Smolock, AR, G Nadolski, M Itkin: Intranodal glue embolization for the management of postsurgical groin lymphocele and lymphorrhea. J. Vasc. Interven. Radiol. 29 (2018), 1462-1465. 10.1016/j.jvir.2018.04.020.

44. Majdalany, BS, G El-Haddad: Contemporary lymphatic interventions for post-operative lymphatic leaks. Trans. Androl. Urol. 9(Suppl 1), (2020), S104-S113. https://doi.org/10.21037/tau.2019.08.15.

45. Keskin, MS, ÖB Argun, C Öbek, et al: The incidence and sequela of lymphocele formation after robot-assisted extended pelvic lymph node dissection. BJU Int. 118 (2016), 127-131. 10.1111/bju.13425.

46. Cohan, RH, M Saeed, SK Sussman, et al: Percutaneous drainage of pelvic lymphatic fluid collections in the renal transplant patient. Invest. Radiol. 22 (1987), 864-867. 10.1097/00004424-198711000-00004.

47. Nezami, N, H Jarmakani, A Marino, et al: Lipiodol-based lymphangiography and gluebased embolization of retroperitoneal lymphatic vessels to treat symptomatic retroperitoneal lymphocele: A case report. Clinics in Surgery (2018), Article 2131.

Nariman Nezami, MD

22 S. Greene Street

University of Maryland Medical Center

Baltimore, MD 21201 USA

E-mail: dr.nezami@gmail.com 